\title{
1 Adaptation of the autosomal part of the genome on the presence of dioecy
}

3 Jitka Žlůvová*, Roman Hobza, Bohuslav Janoušek*

4 Department of Plant Developmental Genetics, Institute of Biophysics, Academy of Sciences

5 of the Czech Republic, Královopolská 135, Brno, Czech Republic.

$6 *$ corresponding authors

7 Email: jitka@ibp.cz, janousek@ibp.cz

8 Author Contributions: J.Z. and B.J. designed research; R.H. contributed new analysis tools;

9 J.Z. and B.J. analyzed data; R.H. discussed the paper; and J.Z. and B.J. wrote the paper.

10 Competing Interest Statement: The authors declare no competing interest.

11 Keywords: genome evolution, adaptive evolution, dioecy

12 This file includes:

13 Main Text

14 Figure 1

15 Table 1 


\section{Abstract}

17 We have attempted to answer the question of whether the presence of sex chromosomes in

18 the genome can affect the evolution of the autosomal part of the genome. As a model, we

19 used dioecious plants from the section Otites of the genus Silene. We have observed a rise in

20 adaptive evolution in the autosomal and pseudoautosomal parts of the genome, which are

21 associated with the evolution of dioecy. This rise is caused neither by the accumulation of

22 sexually antagonistic genes in the pseudoautosomal region nor by the co-evolution of genes

23 acting in mitochondria (in spite of the fact that the dioecy evolved in this case most likely

24 from cytoplasmic male sterility). Thus, this rise in the amount of positively selected codons is

25 most likely caused by the adaptive evolution of genes involved in the specialization of the

26 autosomal part of the genome on the dioecy as described in sex-allocation theory. 


\section{Introduction}

29 Separate sexes are very common in animals, but they also appear in plants (Renner \& Müller, 30 2021). After sex determining gene(s) appear in the genome, many evolutionary processes are 31 started on the sex chromosomes (Charlesworth, 2019). Moreover, many autosomal genes 32 express in a sex-specific manner, and their expression is regulated by the presence of sex33 determining gene(s). These sex-specific differences in gene expression lead to the formation 34 of male and female individuals. But can the presence of separate sexes lead to evolutionary 35 changes in the sequences of autosomal genes? Because males invest more in the quantity of 36 their progeny, whereas the goal of the females is to invest in the quality of the progeny, the 37 presence of separate sexes could lead to the adaptation of the autosomal genes to males' and 38 females' different breeding strategies.

39 In animals, separate sexes and sex chromosomes are of very old origin, and thus it could be 40 very difficult to search for signs of the adaptation of the autosomal part of the genome to the 41 presence of separate sexes. On the other hand, many plant genera contain recently evolved 42 dioecious clades. One of the plant genera with young sex chromosomes is the genus Silene.

43 The dioecious Silene species are especially well-suited to this study because closely related 44 non-dioecious species are known, transcriptomic data for both the dioecious species and their 45 non-dioecious relatives are known, and genetic maps are available in several species 46 (reviewed in (Balounova et al., 2019) ). In the genus Silene, apart from the dosage 47 compensation studies (Martin et al., 2019; Muyle et al., 2018), adaptation of the 48 transcriptome to the dioecy was also studied from a quantitative point of view (Zemp, 49 Widmer, \& Charlesworth, 2018). However, it is not known whether the presence of sex 50 chromosomes in the genome can affect the evolution of the coding regions in the autosomal 51 part of the genome.

\section{Results and discussion}

54 In this study, we use the currently available RNAseq data obtained in the dioecious section

55 Otites to study the influence of the rise of dioecy on the evolution of the sequences that are 56 not sex-linked. Results of the phylogenetic analysis shown in Fig. 1 are mostly in accordance 57 with the results from the previous study that did not include S. nocturna and S. paradoxa. A 58 minor difference in topology is that the current results support the clade joining two members 
59 of the group Cyri (defined previously (Balounova et al., 2019) according to phenotypic 60 markers), while in our previous study, the group Cyri appeared as completely polyphyletic

61 (Balounova et al., 2019). Current dating, based on the estimation of the synonymous

62 divergence of the outgroup (S. nocturna) from the other species, suggests the age of dioecious

63 section Otites cca 2.3 million years which is in the range of the previous estimate based on

64 calibration by fossils (1.17-2.60 million years) (Slancarova et al., 2013).

65 The $\omega$ ratio $(\omega=\mathrm{dN} / \mathrm{dS})$ is one of the most widely used statistical tests used to quantify 66 selection pressures acting on protein-coding regions. This measure quantifies selection 67 pressures by comparing the rate of substitutions at silent sites (dS), which are presumed 68 neutral, to the rate of substitutions at non-silent sites $(\mathrm{dN})$, which possibly experience 69 selection. The comparison of the two- and three-ratios branch models in the CODEML 70 program of PAML package (Yang, 2007) shows that the $\omega$ value of the branch preceding sex 71 chromosome evolution ( $\omega=0.16$; blue in Fig. 1) is lower than the $\omega$ value of the branch 72 where dioecy and sex chromosomes evolved $(\omega=0.29$; red in Fig. 1). This difference is 73 highly significant $\left(\mathrm{P}<10^{-99}\right.$, likelihood ratio test; LRT). After the formation of the dioecy 74 (green in Fig. 1), the $\omega$ value significantly decreased $\left(\omega=0.26 ; \mathrm{P}<10^{-99}\right.$, LRT).

75 Because the increase of the $\omega$ values can be caused by the changes in the number of either 76 positively or neutrally selected sites, we compared branch-site models of these branches. The 77 results are summarized in Table 1 . The branch where dioecy evolved showed a significant 78 percentage of positively selected codons $\left(\mathrm{P}<10^{-99}\right.$, LRT). On the other hand, we did not 79 detect codons under positive selection in the branch preceding dioecy (blue in Fig. 1) (P > 80 0.99, LRT). Moreover, the $\omega$ values of sites under purifying selection did not increase 81 significantly $(\mathrm{P}=0.95$, Wilcoxon test). To confirm these results, we performed tests for 82 relaxed selection using RELAX program (Wertheim, Murrell, Smith, Kosakovsky Pond, \& 83 Scheffler, 2015) of HyPhy package (Kosakovsky Pond et al., 2020; Pond, Frost, \& Muse, 84 2005). Transition from the gynodioecy to the dioecy was connected with the selection 85 intensification $(\mathrm{P}=0.00 ; \mathrm{K}=1.55)$. On the other hand, when the branch involving dioecy 86 evolution and the branch after sex chromosome evolution were compared, non-significant 87 relaxation was detected $(\mathrm{P}=0.339, \mathrm{~K}=0.91)$. Most of the identified adaptively evolved 88 codons (63 out of 87 codons) are recruited from the neutrally evolved codons, which is 89 significantly more than by chance ( $\mathrm{P}<10^{-16}$, chi-squared goodness of fit test). Because the 90 dioecy in the Silene section Otites most likely evolved from the gynodioecy, and because the 91 gynodioecy is in the genus Silene usually of nucleo-cytoplasmic type, the dioecy evolved, in 
92 this case, most likely by the genetic fixation of a male sterile cytoplasm and subsequent

93 recruitment of a fertility restorer as sex determining gene, as discussed by (Zluvova et al.,

94 2005). In this case, no drastic change in population size or rate of inbreeding is necessary to

95 open the route to dioecy. The observed absence of any sign of selective pressure relaxation is

96 in accordance with this hypothesis.

97 The rise of the amount of positively selected codons on the branch including sex chromosome

98 evolution cannot be caused by the selection of sexually antagonistic genes on the sex

99 chromosomes (Rice, 1996), because the analysed dataset does not contain any completely

100 sex-linked gene. Moreover, it cannot be caused by the accumulation of sexually antagonistic

101 genes in the pseudoautosomal region, because we found that only one of the studied ESTs

102 was located in this part of the sex chromosomes.

103 Because all close relatives of the analyzed species are gynodioecious, most likely with 104 cytoplasmic male sterility, one plausible explanation for the rise of adaptive evolution can be 105 connected with the presumed nucleo-cytoplasmic origin of the sex-determining system. The 106 fixation of a certain type of male-sterility causing cytoplasm could influence the evolution of 107 nuclear genes involved in mitochondrial metabolism. However, among the 42 ESTs 108 identified as having at least one adaptively evolved codon on the branch involving sex 109 chromosome formation, we did not find an overrepresentation of proteins located in 110 mitochondria (7 ESTs; P = 0.77, Pearson's chi-squared test).

111 We can hypothesize that this rise in the amount of positively selected codons is caused by the 112 selection of genes involved in the specialization of the genome on the dioecy as described in 113 the sex-allocation theory (Charnov, 1982), which states that female plants allocate more 114 resources to the quality of seeds whereas male plants allocate resources into the amount of 115 pollen. However, based on phenotypic data, it is possible to conclude that the adaptation of 116 the genome to the dioecy is rather complex and includes a wide variety of genes (Geber, 117 Dawson, \& Delph, 1999). Indeed, the genes identified as adaptively evolving on the branch 118 where dioecy evolved (see Supporting Information) show rather diverse characteristics. 119 These results are in good accordance with the previous observations on the phenotypic level 120 (Geber et al., 1999).

121 The discovered coincidence between the increased amount of adaptively evolved codons in 122 autosomes and the evolution of dioecy cannot prove causality in this process. However, the 
hypothesis that the changes in autosomes represent an adaptation to the sexually dimorphic phenotype of dioecious species appears most likely.

\section{Materials and methods}

The dataset from the previous work (Balounova et al., 2019) was supplemented by $S$. nocturna (SRR6040876) and S. paradoxa (SRR999296-SRR999299, publically available) samples. S. pseudotites has been excluded from the dataset based on its suspected hybrid origin. The reads of the $S$. nocturna and $S$ paradoxa were assembled using Trinity (Haas et al., 2013). The assembly was treated with an Evigene pipeline to reduce the level of duplicates and used as a reference. The reads were mapped using Bowtie2 (Langmead \& Salzberg, 2012), the SNPs were called via FreeBayes (Garrison \& Marth, 2012) and phasing was performed with WhatsHap (Patterson et al., 2015). The regions with coverage lower than 2 masked using the maskfasta method in BEDTools (Quinlan \& Hall, 2010). The phased sequences were added to the original alignments using the MAFFT aligner (Katoh \& Standley, 2013) based on the results of best reciprocal blast hit search (Camacho et al., 2009). Phylogenetic analysis was done using the StarBeast2 module in BEAST 2 (Bouckaert et al., 2014; Ogilvie, Bouckaert, \& Drummond, 2017). Two independent chains were run for 1000 000000 states (Yule model, birth-death). For the dating, the calibration of the most recent common ancestor of S. nocturna and the other species was done similarly to Balounova et al. (Balounova et al., 2019) (based on estimated divergence per synonymous site dS (substitutions per synonymous site) and the divergence time estimates for several angiosperm lineages (Brassicaceae, Malvaceae, Euphorbiaceae, Fabaceae, Cucurbitaceae, Rosaceae, Solanaceae, and Poaceae), so as not to depend on a single fossil record or phylogenetic tree position, which yielded a mean substitution rate of $5.35 \times 10^{-9}$ synonymous substitutions/site/ year) (De La Torre, Li, Van de Peer, \& Ingvarsson, 2017). Ks values for the distance of individual species of the section Otites to S. nocturna were estimated using KaKs calculator 2 program (Wang, Zhang, Zhang, Zhu, \& Yu, 2010), and the mean value was used for the calibration of the tree. The resulting dataset did not contain any of the completely sex-linked genes identified previously (Balounova et al., 2019) (Martin et al., 2019).

PAML analyses were used to determine whether some ESTs evolved under selective pressure. The CODEML program of PAML (Yang, 2007) was used to estimate the ratio $(\omega)$ of the non-synonymous substitution rate $(\mathrm{dN})$ to the synonymous substitution rate $(\mathrm{dS})$. As 
155 the reference tree, the phylogenetic tree constructed as described above was used. The

156

157

158

159

160

161

162

163

164

165

166

167

168

169

170

171

173

\section{Acknowledgments}

184 This research was supported by the Czech Science Foundation (grant 19-15609S).

185 Computational resources were supplied by the project "e-Infrastruktura CZ" (e-INFRA 186 LM2018140) provided within the program Projects of Large Research, Development and 
187 Innovations Infrastructures and by the ELIXIR-CZ project (LM2018131), part of the 188 international ELIXIR infrastructure.

189

\section{Competing Interest Statement}

191 No financial and/or non-financial competing interests declared. 


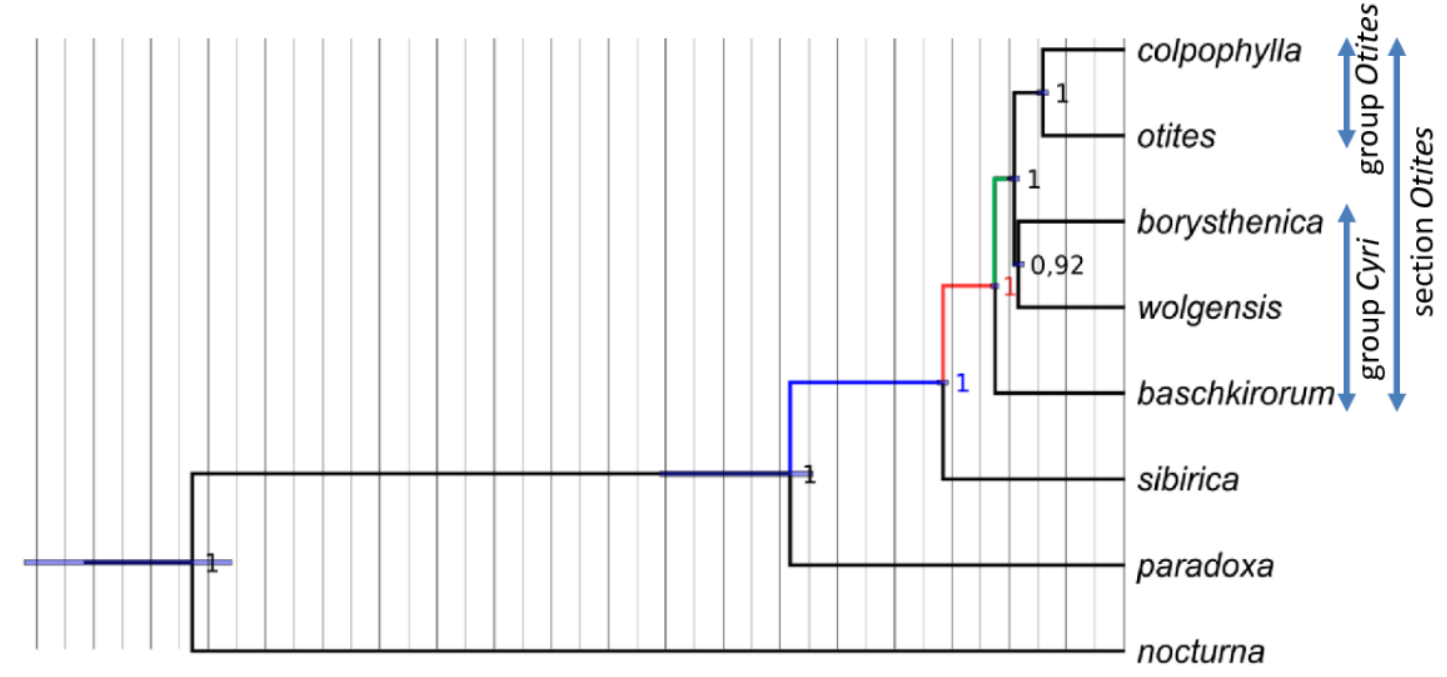

198 colors of the highlighted branches refer to analyses of adaptive analyses. For details, see

199 Results and Discussion. 


\begin{tabular}{|c|c|c|c|c|c|c|}
\hline \multirow[b]{2}{*}{ branch } & \multicolumn{2}{|c|}{$\omega<1$} & \multicolumn{2}{|c|}{$\omega=1$} & \multicolumn{2}{|c|}{$\omega>1$} \\
\hline & $\begin{array}{c}\text { percentage } \\
(\%)\end{array}$ & $\begin{array}{c}95 \% \mathrm{Cl} \text { of } \\
\text { percentage } \\
\text { difference }(\%)\end{array}$ & $\begin{array}{c}\text { percentage } \\
(\%)\end{array}$ & $\begin{array}{c}95 \% \mathrm{Cl} \text { of } \\
\text { percentage } \\
\text { difference }(\%)\end{array}$ & $\begin{array}{l}\text { percentage } \\
(\%)\end{array}$ & $\begin{array}{c}95 \% \mathrm{Cl} \text { of } \\
\text { percentage } \\
\text { difference }(\%)\end{array}$ \\
\hline \multirow[t]{2}{*}{$\begin{array}{l}\text { preceding sex } \\
\text { chromosome evolution }\end{array}$} & 80.496 & & 19.504 & & 0 & \\
\hline & & $-0.19-0.31$ & & $-0.17-0.34$ & & $0.02-0.03$ \\
\hline \multirow[t]{2}{*}{$\begin{array}{l}\text { including sex } \\
\text { chromosome evolution }\end{array}$} & 80.557 & & 19.419 & & 0.024 & \\
\hline & & $-0.2-0.31$ & & $-0.17-0.32$ & & $0.02-0.03$ \\
\hline $\begin{array}{l}\text { following sex } \\
\text { chromosome evolution }\end{array}$ & 80.497 & & 19.503 & & 0 & \\
\hline
\end{tabular}

203 Table 1. Branch-site analysis of Silene transcriptome before and after sex chromosome

204 evolution. Confidence intervals (CI) are given for the difference of percentage between the

205 neighboring rows. Statistically significant values are highlighted in bold. 
206

207

208

209

210

211

212

213

214

215

216

217

218

219

220

221

222

223

224

225

226

227

228

229

230

\section{Bibliography}

Balounova, V., Gogela, R., Cegan, R., Cangren, P., Zluvova, J., Safar, J., ... Janousek, B. (2019). Evolution of sex determination and heterogamety changes in section Otites of the genus Silene. Scientific Reports, 9(1), 1045. doi: 10.1038/s41598-018-37412-x

Bouckaert, R., Heled, J., Kühnert, D., Vaughan, T., Wu, C.-H., Xie, D., ... Drummond, A. J. (2014). BEAST 2: a software platform for Bayesian evolutionary analysis. PLoS Computational Biology, 10(4), e1003537. doi: 10.1371/journal.pcbi.1003537

Camacho, C., Coulouris, G., Avagyan, V., Ma, N., Papadopoulos, J., Bealer, K., \& Madden, T. L. (2009). BLAST+: architecture and applications. BMC Bioinformatics, 10, 421. doi: $10.1186 / 1471-2105-10-421$

Charlesworth, D. (2019). Young sex chromosomes in plants and animals. The New Phytologist, 224(3), 1095-1107. doi: 10.1111/nph.16002

Charnov, E. L. (1982). The theory of sex allocation. Monographs in Population Biology, 18, $1-355$.

De La Torre, A. R., Li, Z., Van de Peer, Y., \& Ingvarsson, P. K. (2017). Contrasting Rates of Molecular Evolution and Patterns of Selection among Gymnosperms and Flowering Plants. Molecular Biology and Evolution, 34(6), 1363-1377. doi: 10.1093/molbev/msx069

Garrison, E., \& Marth, G. (2012). Haplotype-based variant detection from short-read sequencing.

Geber, M. A., Dawson, T. E., \& Delph, L. F. (Eds.). (1999). Gender and sexual dimorphism in flowering plants. Berlin, Heidelberg: Springer Berlin Heidelberg. doi: 10.1007/978-3662-03908-3

Haas, B. J., Papanicolaou, A., Yassour, M., Grabherr, M., Blood, P. D., Bowden, J., ... Regev, A. (2013). De novo transcript sequence reconstruction from RNA-seq using the 
Trinity platform for reference generation and analysis. Nature Protocols, 8(8), 1494-

Katoh, K., \& Standley, D. M. (2013). MAFFT multiple sequence alignment software version 772-780. doi: 10.1093/molbev/mst010

Kosakovsky Pond, S. L., Poon, A. F. Y., Velazquez, R., Weaver, S., Hepler, N. L., Murrell,

Langmead, B., \& Salzberg, S. L. (2012). Fast gapped-read alignment with Bowtie 2. Nature Methods, 9(4), 357-359. doi: 10.1038/nmeth.1923 Distinct Sex Determination Systems. Genome Biology and Evolution, 11(2), 350-361. doi: $10.1093 /$ gbe/evz001

Muyle, A., Zemp, N., Fruchard, C., Cegan, R., Vrana, J., Deschamps, C., ... Marais, G. A. B. (2018). Genomic imprinting mediates dosage compensation in a young plant XY system. Nature Plants, 4(9), 677-680. doi: 10.1038/s41477-018-0221-y

Newcombe, R. G. (1998). Two-sided confidence intervals for the single proportion: comparison of seven methods. Statistics in Medicine, 17(8), 857-872. doi: 10.1002/(sici)1097-0258(19980430)17:8<857::aid-sim777>3.0.co;2-e

Ogilvie, H. A., Bouckaert, R. R., \& Drummond, A. J. (2017). Starbeast2 brings faster species tree inference and accurate estimates of substitution rates. Molecular Biology and Evolution, 34(8), 2101-2114. doi: 10.1093/molbev/msx126

Patterson, M., Marschall, T., Pisanti, N., van Iersel, L., Stougie, L., Klau, G. W., \& 
Pond, S. L. K., Frost, S. D. W., \& Muse, S. V. (2005). HyPhy: hypothesis testing using phylogenies. Bioinformatics, 21(5), 676-679. doi: 10.1093/bioinformatics/bti079

Quinlan, A. R., \& Hall, I. M. (2010). BEDTools: a flexible suite of utilities for comparing genomic features. Bioinformatics, 26(6), 841-842. doi: 10.1093/bioinformatics/btq033 expanding recombination suppression and genetic degeneration. Nature Plants, 7(4), 392-402. doi: 10.1038/s41477-021-00884-3

Rice, W. R. (1996). Sexually antagonistic male adaptation triggered by experimental arrest of female evolution. Nature, 381(6579), 232-234. doi: 10.1038/381232a0 females in silene. Evolution, 67(12), 3669-3677. doi: 10.1111/evo.12223

Wang, D., Zhang, Y., Zhang, Z., Zhu, J., \& Yu, J. (2010). KaKs_Calculator 2.0: a toolkit incorporating gamma-series methods and sliding window strategies. Genomics, Proteomics \& Bioinformatics / Beijing Genomics Institute, 8(1), 77-80. doi: $10.1016 / \mathrm{S} 1672-0229(10) 60008-3$ and Evolution, 32(3), 820-832. doi: 10.1093/molbev/msu400 
281 Yang, Z. (2007). PAML 4: phylogenetic analysis by maximum likelihood. Molecular Biology and Evolution, 24(8), 1586-1591. doi: 10.1093/molbev/msm088

283 Zemp, N., Widmer, A., \& Charlesworth, D. (2018). Has adaptation occurred in males and

284 females since separate sexes evolved in the plant Silene latifolia? Proceedings.

285 Biological Sciences / the Royal Society, 285(1883). doi: 10.1098/rspb.2017.2824

286 Zluvova, J., Lengerova, M., Markova, M., Hobza, R., Nicolas, M., Vyskot, B., ... Janousek, B. (2005). The inter-specific hybrid Silene latifolia x S. viscosa reveals early events of sex chromosome evolution. Evolution \& Development, 7(4), 327-336. doi: 